\title{
On community detection in very large networks
}

\author{
Alexandre P. Francisco and Arlindo L. Oliveira \\ INESC-ID / CSE Dept, IST, Tech Univ of Lisbon \\ Rua Alves Redol 9, 1000-029 Lisboa, PT \\ $\{$ aplf, aml $\}$ @inesc-id.pt
}

\begin{abstract}
Community detection or graph clustering is an important problem in the analysis of computer networks, social networks, biological networks and many other natural and artificial networks. These networks are in general very large and, thus, finding hidden structures and functional modules is a very hard task. In this paper we propose new data structures and a new implementation of a well known agglomerative greedy algorithm to find community structure in large networks, the CNM algorithm. The experimental results show that the improved data structures speedup the method by a large factor, for large networks, making it competitive with other state of the art algorithms.
\end{abstract}

\section{Introduction}

The problem of graph clustering or community finding has been extensively studied and, for the majority of the interesting formulations, this problem is NP-hard. Thus, in the study of large networks, fast approximation algorithms are required even though we may obtain suboptimal solutions. For a deep review on this topic, we refer the reader to a recent survey on community finding by Fortunato [1]. Here we revisit the modularity maximization problem, which is NP-hard [2], and a well known greedy approach proposed by Newman [3]. The simplest algorithm based on his approach runs in $O(n(n+m))$ time, or $O\left(n^{2}\right)$ for sparse graphs, where $n$ is the number of vertices and $m$ is the number of edges. More recently, Clauset et al. [4] exploited some properties of the optimization problem and, by using more sophisticated data structures, they proposed the $C N M$ algorithm which runs in $O(m d \log n)$ time in the worst case, where $d$ is the depth of the dendrogram that describes the community structure.

In this paper we propose a new implementation of the CNM algorithm, using improved data structures. Although the asymptotic time bound is the same of the CNM algorithm, experimental results show a speed up of at least a factor of two. Moreover, we introduced randomization within our implementation which is useful to evaluate stability as different runs can provide different clusterings. The experimental evaluation includes several public available datasets and benchmarks. We also evaluate the performance of our implementation on large graphs generated with the partial duplication model [5]. The maximum modularity values obtained for these graphs are rather large, which is interesting given that these are random graphs. Finally, we briefly discuss the application and integration of this method with other measures and schemata. 


\section{Algorithm and data structures}

The proposed algorithm starts with each vertex being the sole member of its community and then, iteratively, it merges pairs of communities that maximize the modularity score $Q$. Given a graph and a specific division of it into communities, modularity evaluates the difference between the fraction of edges that fall within communities and the expected fraction of edges within communities, if the edges were randomly distributed while respecting vertices degrees [6]. Let $G=(V, E)$ be an undirected graph and $A$ its adjacency matrix, i.e., $A_{u v}=1$ if $(u, v) \in E$, and $A_{u v}=0$ otherwise. Let $n$ be the number of vertices and $m$ be the number of edges of $G$. The degree $d_{u}$ of a vertex $u \in V$ is given by $\sum_{v \in V} A_{u v}$. A clustering or partition $\mathcal{P}$ of $G$ is a collection of sets $\left\{V_{1}, \ldots, V_{k}\right\}$, with $k \in \mathbb{N}$, such that $V_{i} \neq \emptyset$, for $1 \leq i \leq k, V_{i} \cap V_{j}=\emptyset$, for $1 \leq i<j \leq k$, and $\bigcup_{1 \leq i \leq k} V_{i}=V$. Given a partition $\mathcal{P}$ for $G$, we compute its modularity as

$$
Q(\mathcal{P})=\frac{1}{2 m} \sum_{u, v \in V}\left[A_{u v}-\frac{d_{u} d_{v}}{2 m}\right] \delta_{\mathcal{P}}(u, v)
$$

where $m=|E|$ and the $\delta_{\mathcal{P}}$-function is such that $\delta_{\mathcal{P}}(u, v)=1$ if both $u, v \in C$ for some $C \in \mathcal{P}, \delta_{\mathcal{P}}(u, v)=0$ otherwise. The modularity $Q_{G}$ of a graph $G$ is defined as the maximum modularity over all possible graph partitions. Although Eq. (1) can take negative values, $Q_{G}$ takes values between 0 and 1 . Values near 1 indicate strong community structure and 0 is obtained for the trivial partition where all nodes belong to the same community. Typically, values for graphs with known community structure are in the range from 0.3 to $0.7[6,7]$.

Let $C_{i}, C_{j} \in \mathcal{P}_{t}$ be two communities, where $0 \leq i, j<\left|\mathcal{P}_{t}\right|$ and $\mathcal{P}_{t}$ is the partition achieved after $t \geq 0$ iterations. The change $\Delta Q_{i j}$ in $Q$ after merging $C_{i}$ and $C_{j}$ to form a new partition $\mathcal{P}_{t+1}$ is given by manipulation of Eq. (1),

$$
\Delta Q_{i j}=Q\left(\mathcal{P}_{t+1}\right)-Q\left(\mathcal{P}_{t}\right)=\frac{1}{2 m} 2 \sum_{u \in C_{i}} \sum_{v \in C_{j}}\left[A_{u v}-\frac{d_{u} d_{v}}{2 m}\right] .
$$

Since calculating the $\Delta Q_{i j}$ for each pair $C_{i}, C_{j}$ and for each iteration $t$ becomes time-consuming, as in the original CNM algorithm, we store these values for each

pair and only update them when needed. Given $C_{i} \in \mathcal{P}_{t}$, let $\bar{d}_{i}=\sum_{u \in C_{i}} d_{u} /(2 m)$ and assume that $C_{i}, C_{j} \in \mathcal{P}_{t}$ are merged into $C_{k} \in \mathcal{P}_{t+1}$ at iteration $t+1$. Then, for $\mathcal{P}_{t+1}, \bar{d}_{k}=\bar{d}_{i}+\bar{d}_{j}$ and, for each $C_{\ell}$ adjacent to $C_{k}$,

$$
\Delta Q_{k \ell}= \begin{cases}\Delta Q_{i \ell}+\Delta Q_{j \ell} & \text { if } C_{\ell} \text { is connected to } C_{i} \text { and } C_{j}, \\ \Delta Q_{i \ell}-2 \bar{d}_{j} \bar{d}_{\ell} & \text { if } C_{\ell} \text { is connected to } C_{i} \text { but not to } C_{j}, \\ \Delta Q_{j \ell}-2 \bar{d}_{i} \bar{d}_{\ell} & \text { if } C_{\ell} \text { is connected to } C_{j} \text { but not to } C_{i} .\end{cases}
$$

These equations follow easily from Eq. (2). Communities are adjacent or connected if there is at least one edge between them. Note that merging two communities for which there is no connecting edge does not increase $Q$ (when $C_{i}$ is disconnected from $C_{l}$, the first term of Eq. (2) is zero and only the second one remains). Therefore, we will not store the value $\Delta Q$ for such pairs. 

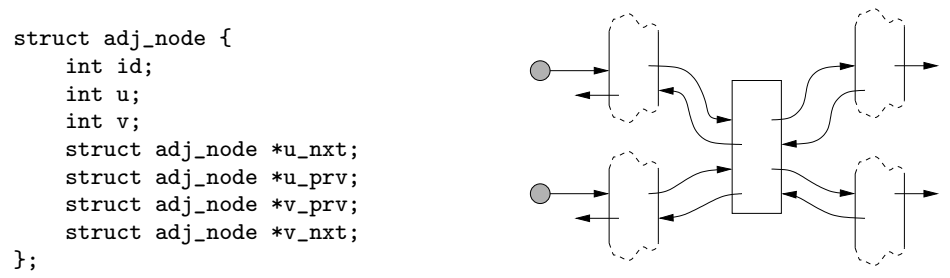

Fig. 1. Cross-linked adjacency list data structure. List nodes are defined by the C structure on the left and are linked as depicted on the right.

As described above, the algorithm starts with each vertex $u \in V$ being the sole member of a cluster. Let $C_{u} \in \mathcal{P}_{0}$ be such that $C_{u}=\{u\}$, for each $u \in$ $V$. Then, for each $u \in V$ and $(u, v) \in E$, we initially set $\bar{d}_{u}=d_{u} / 2 m$ and $\Delta Q_{u v}=1 / m-2 \bar{d}_{u} \bar{d}_{v}$. Accordingly to Eq. (1), the initial value of $Q$ is set to $Q=-\sum_{u \in V} \bar{d}_{u} \bar{d}_{u}$. The algorithm proceeds iteratively as follows:

1. select the pair $(i, j)$ with maximum $\Delta Q_{i j}$;

2. merge $C_{i}$ and $C_{j}$ into $C_{k}$ (assuming that we are in iteration $t+1, \mathcal{P}_{t+1}$ is obtained from $\mathcal{P}_{t}$ replacing $C_{i}$ and $C_{j}$ by $C_{k}$ );

3. update $\bar{d}_{k}$ and $\Delta Q_{k \ell}$ for each $C_{\ell}$ adjacent to $C_{k}$ accordingly to Eq. (3);

4. update modularity $Q$ by adding $\Delta Q_{i j}$;

5. repeat from step 1 until one community remains.

Here we are assuming that the graph is connected and that we are storing each partition $\mathcal{P}_{t}$ obtained at iteration $t$. If the graph is not connected, the algorithm stops when a pair $(i, j)$ does not exist in step 1 . Note also that we are usually interested in the partition that maximizes the modularity score. Thus, we can stop when a pair $(i, j)$, selected in step 1 , is such that $\Delta Q_{i j}<0$. By Eq. (3), we know that $\Delta Q$ values can only decrease after a such pair be selected and, thus, the modularity value will not increase more since all $\Delta Q$ values are negative.

The main point is that we must find the maximum values and extract elements from the adjacency lists as fast as possible. Here we use a single heap data structure to store needed $\Delta Q$ values (at most $m$ ) and cross-linked adjacency lists to store community adjacencies. Since we have to both decrease and increase values in the heap (recall Eq. (3)), we use a binary heap data structure, for which the get maximum operation takes constant time and the insert, delete and update operations take $O(\log m)$ time, in the worst case. For community adjacencies, we use doubly-linked lists with cross references (see Fig. 1) and, thus, we can solve side effects in constant time when merging two adjacency lists.

Let $c_{u}, c_{r}, c_{t}, c_{\ell}$ be real constants. Updating a value in the heap takes $c_{u} \log m$ time and extracting a value takes $c_{r} \log m$. Thus, the extraction in step 1 takes $c_{r} \log m$ time at most and, since there are $m$ elements in the heap, this step is repeated $m$ times at most. Because we get a direct reference in step 1 and we have double-linked lists, removing the edge $(i, j)$ from the community adjacency data structure in step 2 takes constant time. Step 2 requires also $3 c_{\ell} n$ time to merge the adjacencies. Note that there are at most $n$ adjacent communities 
to $C_{i}$ and to $C_{j}$ and that we can solve side effects in constant. Moreover, if a community $C_{k}$ appears twice in the result, we only keep it once. Thus, to achieve linear time with unsorted lists, without loss of generality, we must process the adjacency of $C_{i}$, building a bit array of size $n$ at most, and then process the adjacency of $C_{j}$, checking whenever a community $C_{k}$ occurs in both adjacencies and updating the bit array. Finally we reprocess the adjacency of $C_{i}$ in order to find the communities $C_{k}$ which were not in the adjacency of $C_{j}$. Step 3 is done along with step 2 and each update takes $c_{u} \log m$ time at most. Therefore, step 3 takes less than $c_{u} n \log m$ time. Step 4 takes constant time. Although there exist $m$ elements in the heap, steps $2-4$ are executed at most $n-1$ times and, thus, the running time of the algorithm is at most $c_{r} m \log m+3 c_{\ell} n^{2}+c_{u} n^{2} \log m$, i.e., $O\left(n^{2} \log n\right)$ time in the worst case assuming as usual that $m=O\left(n^{2}\right)$.

The differences between our algorithm and the CNM algorithm reside on how we store $\Delta Q$ values and how we manage the adjacency of the communities. The CNM algorithm stores $\Delta Q$ values in a sparse matrix with each row being stored both as a balanced binary tree and as a binary heap. It maintains also a binary heap containing the largest element of each row. Considering the same maxheap implementation and an efficient implementation of binary trees, updating an element takes $c_{u} \log n$ and extracting an element takes $c_{r} \log n$, where $n$ is the maximum size of the heaps in this case. Thus, step 1 takes $c_{r} \log n$ time. Removing the selected pair from the community adjacency data structure in step 2 takes $2 c_{t} \log n$ to update binary trees plus $2 c_{u} \log n$ to update the heaps. Steps 2 and 3 require also $2 n\left(c_{t}+2 c_{u}\right) \log n+c_{u} n$ time, since we must update the trees, the $k$-th heap and the main heap for each $C_{k}$ in adjacency lists being merged. The heap associated with the resulting adjacency list can be updated in $c_{u} n$ time. Step 4 takes constant time. Since steps $2-3$ are executed at most $n-1$ times, the running time of the CNM algorithm is at most $\left(c_{r}+2 c_{t}\right) n \log n+$ $2\left(c_{t}+2 c_{u}\right) n^{2} \log n+c_{u} n^{2}$, i.e., $O\left(n^{2} \log n\right)$. Note that, although the asymptotic runtime bounds are the same, we get an improvement of at least a factor of two.

For sparse and hierarchical graphs we can provide a better upper bound. A graph $G=(V, E)$ is sparse if $m=O(n)$ and $G$ is hierarchical if the resulting dendrogram for the community merging is balanced. In this case, the sum of the communities degrees at a given depth $d$ is at most $2 \mathrm{~m}$. Therefore the running time is at most $O(m d \log n)$, where $d$ is the depth of the dendrogram. Then for sparse and hierarchical graphs, since $m=O(n)$ and $d=O(\log n)$, the algorithm running time becomes $O\left(n \log ^{2} n\right)$. The space requirement of the algorithm is $O(n+m)$ as we store the connections for each community, a total of at most $n$ communities and $m$ connections, and $m$ elements in the heap.

We also included in our implementation a randomized edge comparison function. As noted by Brandes et al. [2], the algorithm may perform badly if pairs with equal $\Delta Q$ are chosen in some crafted order. Although we cannot avoid undesired behavior by ordering these pairs randomly, we expect that it will not happen frequently. With respect to such fluctuations of modularity, we must mention that even small fluctuations may correspond to very different node clusterings [8]. Thus, several runs may be desirable to evaluate the stability of a given clustering, i.e., how stable is vertex assignment along different runs. 

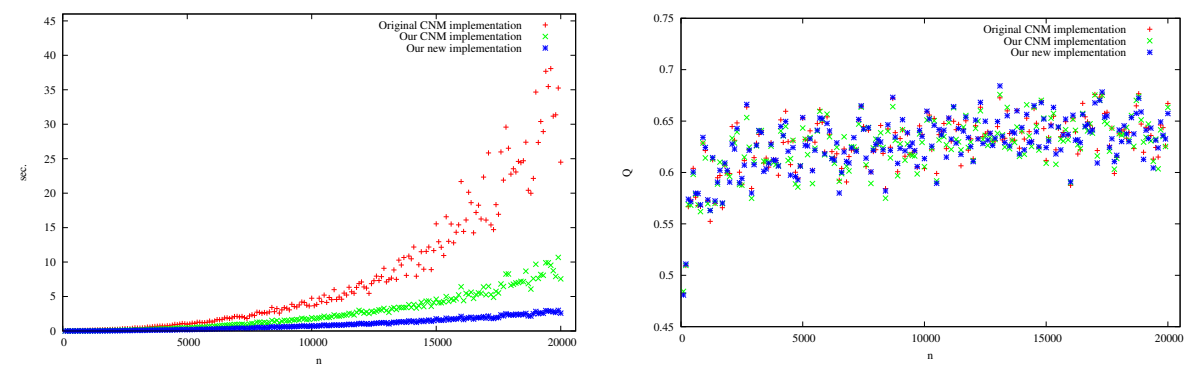

Fig. 2. Average running time and average maximum modularity $Q$ for duplication model graphs obtained with $p=0.5$. For each $n$ were generated 10 random graphs. The number of edges for those graphs is about 10 times the number of vertices.

\section{Experimental evaluation}

In this section we consider 3 implementations in $\mathrm{C}$, the original implementation of the CNM algorithm as provided by the authors, our implementation using optimized data structures to ensure fairness in the comparison and our new implementation. The running times below include the tracking of community membership. For that we use a disjoint sets data structure and, therefore, the running time cost is negligible. All implementations were compiled with the GNU C compiler with flag -03. The experiments were conduced in a $2.33 \mathrm{GHz}$ quad core processor with 16 GB of memory, running a GNU/Linux distribution.

In order to evaluate the performance on large networks, we generated artificial networks from the partial duplication model [5]. Although the abstraction of real networks captured by the partial duplication model, and other generalizations, is rather simple and no community structure is ensured, the global statistical properties of, for instance, biological networks and their topologies can be well represented by this kind of model [9]. For each number of vertices, we generated 10 random graphs with selection probability $p=0.5$, which is within the range of interesting selection probabilities [5]. The number of edges for those graphs is approximately 10 times the number of vertices. Fig. 2 provides the running time of our implementation versus the running time of the CNM algorithm, where we observe an improvement of at least a factor of two. We ran also some tests with very large networks and, for a network with 1 million vertices and more than 13 millions edges, our new implementation takes about 9 hours and requires $744 \mathrm{MB}$ of memory, while our implementation of the CNM algorithm takes 40 hours and requires 1,796 MB. In Section 4 we discuss how prioritizers can further reduce the running time. Although this model does not ensure any community structure, note that the values of modularity are usually higher than 0.5 (see Fig. 2). This is an interesting fact that deserves a better understanding.

Given that in our algorithm we pick randomly a pair whenever two pairs have the same $\Delta Q$ value, we evaluated our implementation on several public 
Table 1. Maximum and minimum modularity for 4 real networks after 1,000 runs. $|V|$ is the number of vertices and $|E|$ is the number of edges for each network. $\max Q$ is the maximum modularity, $\min Q$ is the minimum modularity and $\# \mathcal{P}$ is number of different partitions obtained for 1,000 runs.

\begin{tabular}{lrrrrr} 
Network & $|V|$ & $|E|$ & $\min Q$ & $\max Q$ & $\# \mathcal{P}$ \\
\hline Zachary's karate club [10] & & & & & \\
Bottlenose dolphins'network [11] & 62 & 78 & 0.381 & 0.381 & 1 \\
C. elegans metabolic network [12] & 454 & 2,025 & 0.385 & 0.413 & 253 \\
Protein interaction network [13] & 2,215 & 2,203 & 0.842 & 0.846 & 770
\end{tabular}

datasets and benchmarks, focusing on the stability of the obtained clusterings. Table 1 provides details for four real networks. Unsurprisingly, $Q$ values are identical to those reported by the CNM algorithm. But the partitions found for the last two networks are rather unstable, namely for the protein interaction network where in 1,000 runs 770 different partitions were found. Although we did not analyse further these networks, our results raise an important question concerning partition stability. This is an important issue in the study of networks and, until now, most of the analyses in the literature just consider one partition.

\section{Discussion}

There are alternative approaches for the greedy optimization of modularity. Schuetz and Caflisch [14] proposed an approach where they merge at once $\ell$ disjoint pairs of communities instead of just one pair and which can benefit from improvements proposed here. More recently, Blondel et al. [15] proposed an alternative greedy approach. The algorithm proceeds by alternating two main steps. In the first step, it iteratively considers a vertex, removes it from the current cluster computing the change in modularity, and then selects the cluster that provides the better improvement by moving the vertex to that cluster. This is repeated until no change occurs. The second step consists of building a coarsened graph where each cluster becomes a vertex. Then, we iterate these two steps while there are edges in the coarsened graph. Although the authors do not provide a theoretical bound, the running time seems to be almost linear from the experiments, making it one of the fastest algorithms to date. The improvements proposed in this paper make the CNM algorithm competitive with that algorithm, if not faster. With respect to clustering quality, Noack and Rotta [16] stated that the most effective method consists of a multilevel schema, where the greedy approach studied here is used for the coarsening phase, and the first step of the method proposed by Blondel et al. for the refinement phase. They used also prioritizers to improve both the running time and the clustering quality.

One of the first prioritizers was proposed by Wakita and Tsurumi [17], favoring the merge of equal size communities, enforcing the running time bound of $O\left(n \log ^{2} n\right)$ for sparse graphs. Since Newman and Girvan [6] proposed the 
modularity score to account for the intra-cluster density versus the inter-cluster sparsity, given two clusters or communities $C_{i}$ and $C_{j}$, a natural prioritizer is $\Delta Q_{i j} /\left(d\left(C_{i}\right) d\left(C_{j}\right)\right)$, that favors the merge of clusters with lower weight density, conducting to more dense clusters on average. Although previous studies pointed in this direction [18], only recently Noack and Rotta [16] explicitly used this prioritizer and the variant $\Delta Q_{i j} / \sqrt{d\left(C_{i}\right) d\left(C_{j}\right)}$. Although both prioritizers are related, the second one is closely tied to the null model underlying the modularity measure. By Eq. (2), $2 m \Delta Q_{i j}$ is the difference between the observed and the expected number of edges between $C_{i}$ and $C_{j}$. On the other hand, since the null model assumes a binomial distribution, we know that for large graphs the variance of the number of edges between $C_{i}$ and $C_{j}$ is approximately $d\left(C_{i}\right) d\left(C_{j}\right) /(2 m)$. Thus, the second prioritizer accounts for the number of standard deviations between the observed and the expected number of edges between $C_{i}$ and $C_{j}$. We ran our algorithm with this prioritizer for the network with 13 million edges and we obtained an outstanding speedup, it takes now 130 seconds instead of 9 hours. Also, the values of the modularity did not decrease, as expected, given the modularity definition and its close relation with this prioritizer. As observed before $[19,17]$, the reason for the speedup is that, without any prioritizer, the greedy approach merges the cluster with the largest contribution to modularity with its best neighbor. The strength of the cluster increases and the process continues until all good neighbors are merged. But, since this cluster has great influence, several bad neighbors may also be merged before any other merge pairs be considered. This produces very unbalanced partitions taking the running time up to the upper bound $O\left(n^{2} \log n\right)$. Note also that the modularity value may be lower since some bad neighbors were merged. In fact, after considering the prioritizer, we obtained higher modularity values. For instance, for the Zachary's karate social network we achieved a value of 0.419 . The most important conclusion from our work is that, with the new implementation and considering good prioritizers, we are able to effectively process real large scale-free networks and evaluate its community structure stability.

Several measures have been proposed to evaluate clusterings quality, in particular because modularity suffers some resolution problems [20,21]. Thus, it is important to note that the optimization approach discussed in this paper can be easily adapted for other measures and, in general, it is sufficient to rewrite Eq. (3). This is straightforward for measures based on modularity, such as the modularity for weighted graphs or the similarity-based modularity [22]. For other measures, the updates after each merging step may require some more careful analysis. Nevertheless, we can employ this greedy method either alone or combined with other approaches. For instance, Rosvall and Bergstrom [23, 24] used recently this approach in their study of mutual information and of maps of random walks to uncover community structure. In their work they improve the final results by using a simulated annealing based approach [24].

\section{References}

1. Fortunato, S.: Community detection in graphs. Physics Reports 486 (2010) 75-174 
2. Brandes, U., et al.: On finding graph clusterings with maximum modularity. In: Graph-Theoretic Concepts in Computer Science. Volume 4769 of LNCS., Springer (2007) 121-132

3. Newman, M.E.J.: Fast algorithm for detecting community structure in networks. Physical Review E 69 (2004) 066133

4. Clauset, A., Newman, M.E.J., Moore, C.: Finding community structure in very large networks. Physical Review E 70 (2004) 066111

5. Chung, F., Lu, L., Dewey, T.G., Galas, D.J.: Duplication models for biological networks. Journal of Computational Biology 10(5) (2003) 677-687

6. Newman, M.E.J., Girvan, M.: Finding and evaluating community structure in networks. Physical Review E 69 (2004) 026113

7. Guimerà, R., Sales-Pardo, M., Amaral, L.A.N.: Modularity from fluctuations in random graphs and complex networks. Physical Review E 70(2) (2004) 025101

8. Agarwal, G., Kempe, D.: Modularity-maximizing communities via mathematical programming. The European Physical Journal B 66(3) (2008) 409-418

9. Bhan, A., Galas, D.J., Dewey, T.G.: A duplication growth model of gene expression networks. Bioinformatics 18(11) (2002) 1486-1493

10. Zachary, W.W.: An information flow model for conflict and fission in small groups. Journal of Anthropological Research 33 (1977) 452-473

11. Lusseau, D., et al.: The bottlenose dolphin community of Doubtful Sound features a large proportion of long-lasting associations. Behavioral Ecology and Sociobiology 54(4) (2003) 396-405

12. Duch, J., Arenas, A.: Community identification using extremal optimization. Physical Review E $\mathbf{7 2}$ (2005) 027104

13. Jeong, H., Mason, S., Barabási, A.L., Oltvai, Z.N.: Centrality and lethality of protein networks. Nature 411(6833) (2001) 41-42

14. Schuetz, P., Caflisch, A.: Efficient modularity optimization by multistep greedy algorithm and vertex mover refinement. Physical Review E 77(4) (2008) 46112

15. Blondel, V.D., Guillaume, J.L., Lambiotte, R., Lefebvre, E.: Fast unfolding of communities in large networks. Journal of Statistical Mechanics P10008 (2008)

16. Noack, A., Rotta, R.: Multi-level algorithms for modularity clustering. In: Experimental Algorithms. Volume 5526 of LNCS., Springer (2009) 257-268

17. Wakita, K., Tsurumi, T.: Finding community structure in mega-scale social networks. In: International World Wide Web Conference, ACM (2007) 1275-1276

18. Reichardt, J., Bornholdt, S.: Statistical mechanics of community detection. Physical Review E 74 (2006) 016110

19. Danon, L., Duch, J., Diaz-Guilera, A., Arenas, A.: The effect of size heterogeneity on community identification in complex networks. Journal of Statistical Mechanics P11010 (2006)

20. Fortunato, S., Barthélemy, M.: Resolution limit in community detection. PNAS 104(1) (2007) 36-41

21. Kumpula, J.M., Saramäki, J., Kaski, K., Kertész, J.: Limited resolution in complex network community detection with Potts model approach. The European Physical Journal B - Condensed Matter and Complex Systems 56(1) (2007) 41-45

22. Feng, Z., Xu, X., Yuruk, N., Schweiger, T.A.J.: A novel similarity-based modularity function for graph partitioning. In: Data Warehousing and Knowledge Discovery. Volume 4654 of LNCS., Springer (2007) 385-396

23. Rosvall, M., Bergstrom, C.T.: An information-theoretic framework for resolving community structure in complex networks. PNAS 104(18) (2007) 7327

24. Rosvall, M., Bergstrom, C.T.: Maps of random walks on complex networks reveal community structure. PNAS 105(4) (2008) 111-118 